\title{
Ontology integration to identify protein complex in protein interaction networks
}

\author{
Bo Xu*, Hongfei Lin, Zhihao Yang \\ From International Workshop on Computational Proteomics \\ Hong Kong, China. 18-21 December 2010
}

\begin{abstract}
Background: Protein complexes can be identified from the protein interaction networks derived from experimental data sets. However, these analyses are challenging because of the presence of unreliable interactions and the complex connectivity of the network. The integration of protein-protein interactions with the data from other sources can be leveraged for improving the effectiveness of protein complexes detection algorithms.

Methods: We have developed novel semantic similarity method, which use Gene Ontology (GO) annotations to measure the reliability of protein-protein interactions. The protein interaction networks can be converted into a weighted graph representation by assigning the reliability values to each interaction as a weight. Following the approach of that of the previously proposed clustering algorithm IPCA which expands clusters starting from seeded vertices, we present a clustering algorithm OIIP based on the new weighted Protein-Protein interaction networks for identifying protein complexes.
\end{abstract}

Results: The algorithm OIIP is applied to the protein interaction network of Sacchromyces cerevisiae and identifies many well known complexes. Experimental results show that the algorithm OIIP has higher F-measure and accuracy compared to other competing approaches.

\section{Background}

In the post-genomic era, one of the most important issues is to systematically analyze and comprehensively understand the topology of biological networks and biochemical progress in cells. The current knowledge base of protein-protein interactions has been built from the heterogeneous data sources generated by high-throughput techniques [1-4].Protein complexes can help us to understand certain biological progress and to predict the functions of proteins. A wide range of graphtheoretic approaches have been employed for detecting protein complexes from protein interaction networks. However, they have been limited in accuracy due to the presence of unreliable interactions and the complex connectivity patterns of the networks. The experimental data sets are susceptible to false positives, i.e., some fraction of the

\footnotetext{
* Correspondence: xubo@mail.dlut.edu.cn

Department of Computer Science and Engineering, Dalian University of

Technology, Dalian, China

Full list of author information is available at the end of the article
}

putative interactions detected must be considered spurious because they cannot be confirmed to occur in vivo [5].

To resolve the inaccuracy resulting from false connections, other functional knowledge can be integrated into the protein interaction networks. For example, many groups [6-8] have investigated the integration of gene expression data from microarray experiments to improve protein complexes identification. However, gene expression data are also susceptible to experimental sources of bias and noise. The correlations of mRNA levels with even cognate protein expression may be modest at best. These factors limit the usefulness of microarray data for assessing the reliability of proteinprotein interactions. Gene Ontology (GO) [9] is another useful data source to combine with the protein interaction networks. The GO is currently one of the most comprehensive and well-curated ontology databases in the bioinformatics community. It provides a collection of well-defined biological terms, called GO terms, 
spanning biological processes, molecular functions and cellular components. The GO has been used to facilitate the analysis of gene expression data [10-12].

In this work, we integrate protein-protein interactions with the information content in the GO annotation database and topology weights to enhance the modularization of interaction networks. An unweighted protein interaction network can be converted into a weighted graph representation by assigning a weight to each interaction [13]. The weight of each interaction is interpreted as its reliability, i.e., the probability of the interaction being a true positive. We propose a novel method to measure the reliability of protein-protein interactions using GO annotation data and topology weights. Following the approach of that of the previously proposed clustering algorithm IPCA[14] which expands clusters starting from seeded vertices, we present a clustering algorithm OIIP based on the new large weighted protein interaction networks.

\section{Methods}

\section{Weighted network}

Weights quantify the likelihood of the interaction between every pair of proteins, and they can be estimated by encoding the proteins using gene ontology (GO) consortium. "Ontology" is a specification of a conceptualization that refers to the subject of existence. GO is established by the following three criteria: (I) biological process referring to a biological objective to which the gene or gene product contributes; (II) molecular function defined as the biochemical activity of a gene product; (III) cellular component referring to the place in the cell where a gene product is active. It is very common for the same protein or proteins in the same subfamily to form protein complexes, for example, protein Ste2p and Ste3p from a complex that is among activated $\mathrm{G}$ protein-coupled receptors in yeast cellular mating.[15] It is also common for proteins in heterofamilies to form protein complexes if they share a conservative motif, for example, protein Ctf19, Mcm21, and Okp1 from a heterocomplex in the budding yeast kinetochore.[16] Complicated protein complexes may be formed by multiple proteins, some of which share same biological processes and some are from the same subfamily, for example, Dsl1p complex, involved in GolgiER retrograde transport, includes Dsl1p, Dsl3p, Q/tSNARE proteins, and so forth.[17] Thus GO consortium is considered to be a very helpful vehicle for investigating protein-protein interactions,[18] because these three criteria reflect the attribute of gene, gene product, geneproduct groups and the subcellular localization[19-21].

Semantic similarity has been used in Information Science to evaluate the similarity between two concepts in a taxonomy[22], and we applied it to protein-protein interactions to estimate the similarity between two proteins. Based on the previous method [23], we proposed our semantic similarity method. We define an annotation size of a GO term as the number of annotated proteins on the GO term. The semantic similarity between two proteins is then calculated based on the annotation size of the GO term, on which both proteins are annotated. According to the transitivity property of GO annotation, if a protein $\mathrm{x}$ is annotated on a GO term $\mathrm{g}_{\mathrm{i}}$, it is also annotated on the GO terms on the path from $\mathrm{g}_{\mathrm{i}}$ to the root GO term in the GO structure. Thus, the proportion of the annotation size of a GO term to the total number of annotated proteins can quantify the specificity of the GO term. If two proteins are annotated on a more specific GO term and have more common GO terms, then they are functionally more similar.

Suppose a protein $\mathrm{x}$ is annotated on $\mathrm{m}$ different $\mathrm{GO}$ terms. $S_{i}(x)$ denotes a set of annotated proteins on the $\mathrm{GO}$ term $\mathrm{g}_{\mathrm{i}}$, whose annotation includes $\mathrm{x}$, where $1 \leq \mathrm{i} \leq \mathrm{m}$. In the same way, suppose both $\mathrm{x}$ and $\mathrm{y}$ are annotated on $n$ different $G O$ terms, where $n \leq m . S_{j}(x, y)$ denotes a set of annotated proteins on the GO term $\mathrm{g}_{\mathrm{j}}$, whose annotation includes $x$ and $y$, where $1 \leq j \leq n$. Then, the minimum size of $S_{i}(x), \min _{i}\left|S_{i}(x)\right|$, is less than or equal to $\min _{j}|\operatorname{Sj}(x, y)| . C(x, y)$ denotes the sets of GO terms, whose annotation includes $x$ and $y .|C(x, y)|$ is the number of common GO terms which $x$ and $y$ both have.

Suppose the size of annotation represents the number of annotated proteins on a GO term. Using the annotation size of the most specific GO term, on which two proteins $\mathrm{x}$ and $\mathrm{y}$ are annotated, we define semantic similarity $S_{\text {sem }}(x, y)$ between $x$ and $y$ as follows:

$$
S_{\text {sem }}(x, y)=-|C(x, y)| \times \log \left(\frac{\min _{j}\left|S_{j}(x, y)\right|}{\left|S_{\max }\right|}\right)
$$

$\mathrm{S}_{\max }$ is the maximum size of annotation among all GO terms in a DAG structure. If two proteins $\mathrm{x}$ and $\mathrm{y}$ are annotated on a more specific GO term and more common GO terms than $x$ and $z$, then $x$ is semantically more similar to $y$ than $\mathrm{z}$.

Considering the graph topology, we also involve the topology weight. For an input graph $G=(V, E)$, we assign the topology weight of an edge $[u, v]$ to be the number of neighbors shared by the vertices $u$ and $v$. Then we assigned the sum of $S_{\text {sem }}(u, v)$ and topology weight to the edge between $u$ and $v$ as a weight.

\section{Weighted vertex and selecting seed}

We define the weight of each vertex to be the sum of the weights of its incident edges. After all vertices are assigned weights, we also sort in non-increasing order the vertices by their weights and store them in a queue Sq (vertices of the same weight are ordered in terms of their degrees). 
The complexity of calculating edge weights and vertex weights is $\mathrm{O}(|\mathrm{V}||\mathrm{E}|)$, and the complexity of sorting all vertices by their weights is $\mathrm{O}(|\mathrm{V}| \log |\mathrm{V}|)$.

The notion that vertex weight is a good measure for selecting seeds has been adopted by DPClus [40] and MCODE[24]. Here, we also pick the highest weighted vertices as the seeds. Our procedure proceeds as follows. We pick the first vertex in the queue Sq and use it as a seed to grow a new cluster. Once the cluster is completed, all vertices in the cluster are removed from the queue Sq and we pick the first vertex remaining in the queue Sq as the seed for the next cluster. There is an important difference between this seed selection procedure and the one used in the IPCA algorithm [14]. Our procedure computes the vertex weight for each vertex based on the weighted networks; while the IPCA algorithm computes the vertex weight based on the original networks. We feel that our approach is biologically more meaningful because a complex is not only a dense structure in the original protein network but also have biological function.

\section{Extending cluster}

We introduce a new concept to measure how strongly a vertex $\mathrm{v}$ is connected to a subgraph $\mathrm{K}$ : the interaction probability $\mathrm{E}_{\mathrm{vk}}$ of a vertex $\mathrm{v}$ to a subgraph $\mathrm{K}$, where $\mathrm{v} \notin \mathrm{K}$, is defined by

$$
E_{v k}=\frac{e_{v k}}{w_{k}}
$$

Where $e_{\mathrm{vk}}$ is the sum of the weights of edges between the vertex $v$ and $K$, and $w_{k}$ is the sum of weights of edges in $\mathrm{K}$. We discuss the relationship between the parameter $\mathrm{E}_{\mathrm{vk}}$ and $\mathrm{IN}_{\mathrm{vK}}$ introduced in the algorithm IPCA[14]. According to [14], $\mathrm{IN}_{\mathrm{vK}}$ is defined as $I N_{v K}=\frac{m_{v K}}{n_{K}}$, where $\mathrm{m}_{\mathrm{vK}}$ is the number of edges between the vertex $v$ and $K$, and $n_{K}$ is the number of vertices in $\mathrm{K}$. By the expressions, our parameter $E_{\mathrm{vk}}$ is similar to the parameter $\mathrm{IN}_{\mathrm{vK}}$. While our parameter considers with the biological weights, it have more biological meaning.

A cluster $\mathrm{K}$ is extended by adding vertices recursively from its neighbors according to the priority. The priority of a neighbor $\mathrm{v}$ of $\mathrm{K}$ is determined by the value $\mathrm{E}_{\mathrm{vk}}$. This procedure is similar to the one proposed in IPCA [14], except that we do not use $\mathrm{IN}_{\mathrm{vk}}$ to judge the extending. So whether a high priority vertex $\mathrm{v}$ is added to the cluster is determined by the Extend-judgment test below.

Let $\mathrm{T}_{\text {in }}$ be a threshold ranging between 0 and 1 , let $\mathrm{d}$ be a positive integer, and let $K$ be a subgraph. SP is the shortest path. A vertex $v \notin \mathrm{K}$ is added to the cluster if the following two conditions are satisfied (where $\mathrm{K}+\mathrm{v}$ denotes the subgraph induced by $K$ and $v$ ):

1. $\mathrm{E}_{\mathrm{vk}} \geq \mathrm{T}_{\mathrm{in}}$; and

2. The $(\mathrm{SP}(\mathrm{K}+\mathrm{v}) \leq \mathrm{d})$

Only when the candidate vertex $\mathrm{v}$ is satisfied the conditions, can it be added to the cluster. Once the new vertex $\mathrm{v}$ is added to the cluster, the cluster is updated.

\section{Evaluation}

Before we present the results of our comparative experiments, let us first introduce the various evaluation metrics that have been used to evaluate their computational methods for complex detection. We will then present the experimental results of comparing different state-of-theart techniques using these evaluation metrics.

Overall, there are three types of evaluation metrics used to evaluate the quality of the predicted complexes and compute the overall precision of the prediction methods.

\section{Precision, recall and $\mathrm{f}$-measure}

Precision, recall and F-measure are commonly-used evaluation metrics in information retrieval and machine learning. For evaluating protein complex prediction, we need to define how well a predicted complex which consists of a set of protein members, matches an actual complex, which is another set of protein members. The neighborhood affinity score NA (p, b) between a predicted complex $p=\left(V_{p}, E_{p}\right)$ and a real complex $b=\left(V_{b}\right.$, $\left.E_{b}\right)$ in the benchmark complex set, as defined in equation (3) below, can be used to determine whether they match with each other. If NA $(\mathrm{p}, \mathrm{b}) \geq \omega$, they are considered to be matching ( $\omega$ is usually set as 0.20 or 0.25 ). Let $\mathrm{P}$ and $\mathrm{B}$ denote the sets of complexes predicted by a computational method and real ones in the benchmark, respectively. Let $\mathrm{N}_{\mathrm{cp}}$ be the number of predicted complexes which match at least one real complex and $\mathrm{N}_{\mathrm{cb}}$ be the number of real complexes that match at least one predicted complex. Precision and Recall are then defined as follows: [25-27]

$$
\begin{aligned}
& N A(p, b)=\frac{\left|V_{p} \cap V_{b}\right|^{2}}{\left|V_{p}\right| \times\left|V_{b}\right|} \\
& N_{c p}=|\{p \mid p \in P, \exists b \in B, N A(p, b) \geq \omega\}| \\
& N_{c b}=|\{b \mid b \in B, \exists p \in P, N A(p, b) \geq \omega\}| \\
& \text { Precision }=\frac{N_{c p}}{|P|} \text { Recall }=\frac{N_{c b}}{|B|}
\end{aligned}
$$


F-measure, or the harmonic mean of Precision and Recall, can then be used to evaluate the overall performance

$$
F=2 \times \text { Precision } \times \text { Recall } /(\text { Precision }+ \text { Recall })
$$

\section{Sensitivity, positive predictive value and accuracy}

Recently, sensitivity (Sn), positive predictive value (PPV) and accuracy (Acc) have also been proposed to evaluate the accuracy of the prediction methods $[28,29]$. Given $n$ benchmark complexes and $\mathrm{m}$ predicted complexes, let $\mathrm{T}_{\mathrm{ij}}$ denote the number of proteins in common between $i_{\text {th }}$ benchmark complex and $j_{\text {th }}$ predicted complex. $S n$ and PPV are then defined as follows:

$$
\begin{gathered}
S n=\frac{\sum_{i=1}^{n} \max _{j}\left\{T_{i j}\right\}}{\sum_{i=1}^{n} N_{i}} \\
P P V=\frac{\sum_{j=1}^{m} \max _{i}\left\{T_{i j}\right\}}{\sum_{j=1}^{m} T_{. j}}
\end{gathered}
$$

Here $\mathrm{N}_{\mathrm{i}}$ is the number of proteins in the $\mathrm{i}_{\text {th }}$ benchmark complex.

$$
T_{j}=\sum_{i=1}^{n} T_{i j}
$$

Generally, high Sn values indicate that the prediction has a good coverage of the proteins in the real complexes, while high PPV values indicate that the predicted complexes are likely to be true positives. As a summary metric, the accuracy of a prediction, Acc, can then be defined as the geometric average of sensitivity and positive predictive value,

$$
A c c=\sqrt{S n \times P P V}
$$

\section{P-values (functional homogeneity)}

As we gained more and more biological knowledge about the proteins, we can associate a protein with (possibly multiple) functional annotations. The statistical significance of the occurrence of a protein cluster (predicted protein complex) with respect to a given functional annotation can be computed by the following hypergeometric distribution in equation (12) [30,31]:

$$
P-\text { value }=1-\sum_{i=0}^{k-1} \frac{\left(\begin{array}{c}
|F| \\
i
\end{array}\right)\left(\begin{array}{c}
|V|-|F| \\
|C|-i
\end{array}\right)}{\left(\begin{array}{c}
|V| \\
|C|
\end{array}\right)}
$$

Where a predicted complex $\mathrm{C}$ contains $\mathrm{k}$ proteins in the functional group $\mathrm{F}$ and the whole PPI network contains $|\mathrm{V}|$ proteins. The functional homogeneity of a predicted complex is the smallest p-value over all the possible functional groups. A predicted complex with a low functional homogeneity indicates it is enriched by proteins from the same function group and it is thus likely to be true protein complex. By setting a common threshold which specifies the acceptable level of statistical significance, the numbers of predicted complexes with functional homogeneity under this threshold for the various methods can then be used for evaluating their respective overall performance.

\section{Results and discussion}

The protein interaction database is downloaded from the Gavin database [31] and BioGrid (version yeast HCBIOGRID-2.0.31). The protein-complex dataset CYC2008 [38] which we used is a comprehensive catalogue of 408 manually curated heterometic protein complexes reliably backed by small-scale experiment reported. We apply the proposed algorithm OIIP to this two databases. In the following subsections, we discuss the effect of the value $T_{\text {in }}$ on clustering, compare the predicted clusters with the known complexes, evaluate the significance of the predicted clusters. We will also compare the algorithm OIIP to eight competing previous methods for their performance of identifying protein complexes. Since most proteins in the same complex have same or correlative function and involve in the same biological process, we employ biological annotation information, including Go cellular component annotation [39], GO Molecular Function annotation [39] and GO Biological process annotation [39] to assess the predicted protein-complexes.

\section{The effect of $\mathrm{T}_{\text {in }}$ on clustering}

To understand how the value of $T_{\text {in }}$ influences the outcome of the clustering, we generate 9 sets of clusters by using $\mathrm{T}_{\text {in }}=0.1,0.2, \ldots, 0.9$ from the Gavin PPI dataset. The effect on the predicted clusters with different $T_{\text {in }}$ is given in Figure 1.

Figure 1(a) shows that the total number of the predicted clusters is increasing as $T_{\text {in }}$ increases. However, there is a abrupt decrease at $T_{\text {in }}=0.5$. This is probably caused by the Hub structures in the protein interaction 

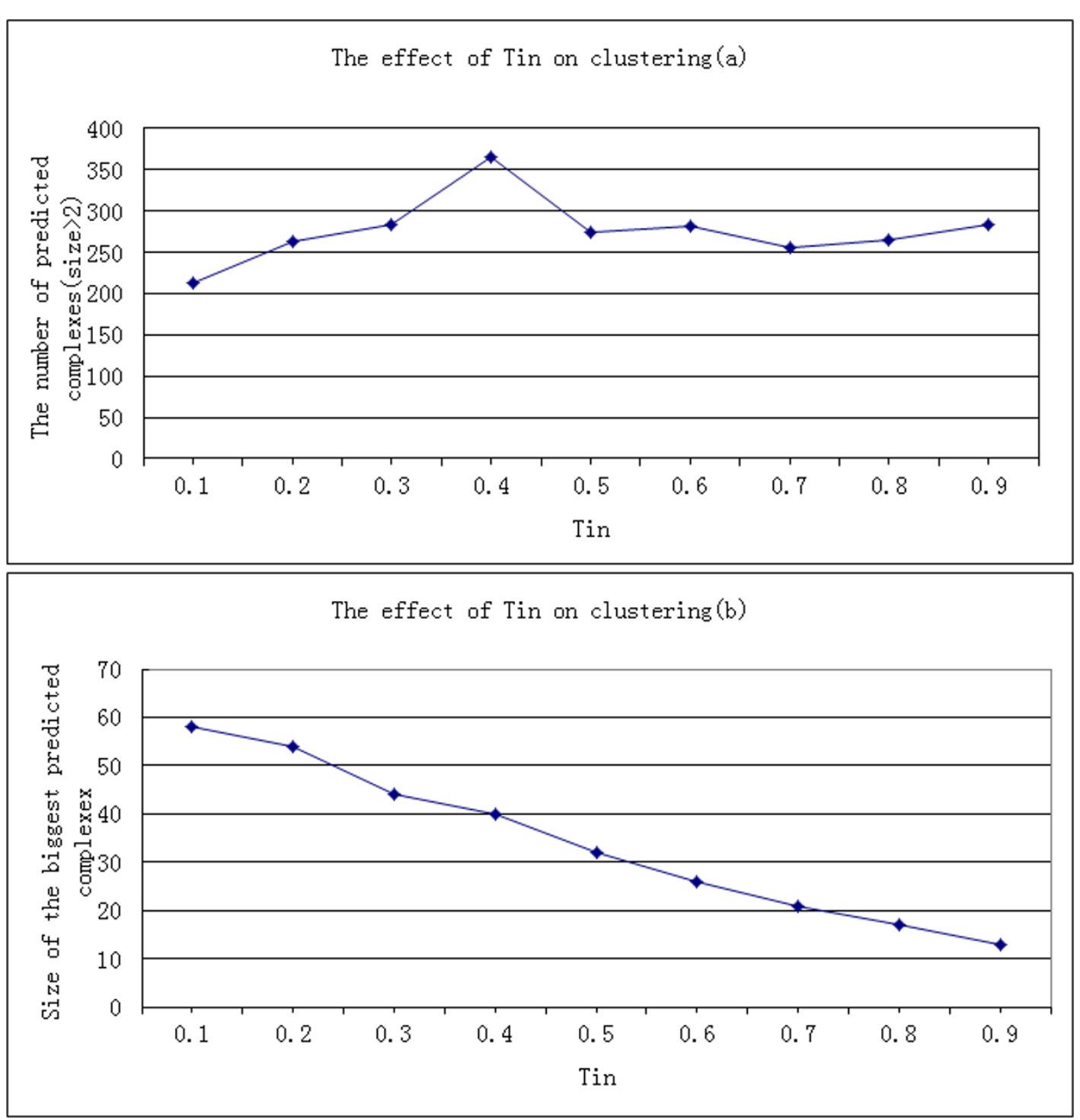

Figure 1 The effect of $\mathbf{T}_{\text {in }}$ on clustering. The effect on the predicted clusters with different $T_{\text {in }}$ is given in this Figure 1. Figure 1 (a) shows that the total number of the predicted clusters is increasing as $T_{\text {in }}$ increases. However, there is an abrupt decrease at $T_{\text {in }}=0.5$. Figure $1(\mathrm{~b})$ shows that the size of the biggest cluster is decreasing as $T_{\text {in }}$ increases. With the increasing of $T_{i n}$ the probability of neighbors added to the cluster is decreasing. Thus, the size of the predicted clusters is also decreasing.

Table 1 The Precision, Recall, F-measure, sensitivity, PPV and Accuracy of the predicted complexes by OIIP using different parameters

\begin{tabular}{ccccccc}
\hline Parameter & Precision & Recall & F-measure & sensitivity & PPV & Accuracy \\
\hline $\mathrm{T}_{\text {in }}=0.1$ & 0.4819 & 0.335784 & 0.395787 & 0.525 & 0.465604 & 0.494491 \\
$\mathrm{~T}_{\text {in }}=0.2$ & 0.498113 & 0.352941 & 0.413146 & 0.511979 & 0.48535 & 0.498487 \\
$\mathrm{~T}_{\text {in }}=0.3$ & 0.482112 & 0.365196 & 0.415588 & 0.501042 & 0.516012 & 0.508472 \\
$\mathrm{~T}_{\text {in }}=0.4$ & 0.559816 & 0.384804 & 0.456097 & 0.484896 & 0.560703 & 0.521424 \\
$\mathrm{~T}_{\text {in }}=0.5$ & 0.606195 & 0.335784 & 0.432177 & 0.420312 & 0.57216 & 0.490394 \\
$\mathrm{~T}_{\text {in }}=0.6$ & 0.582645 & 0.340686 & 0.429963 & 0.409375 & 0.621008 & 0.504207 \\
$\mathrm{~T}_{\text {in }}=0.7$ & 0.485768 & 0.343137 & 0.402181 & 0.386458 & 0.638547 & 0.496761 \\
$\mathrm{~T}_{\text {in }}=0.8$ & 0.458477 & 0.338235 & 0.389283 & 0.360417 & 0.653266 & 0.48523 \\
$\mathrm{~T}_{\text {in }}=0.9$ & 0.466227 & 0.345588 & 0.396944 & 0.340625 & 0.663043 & 0.475236 \\
\hline
\end{tabular}



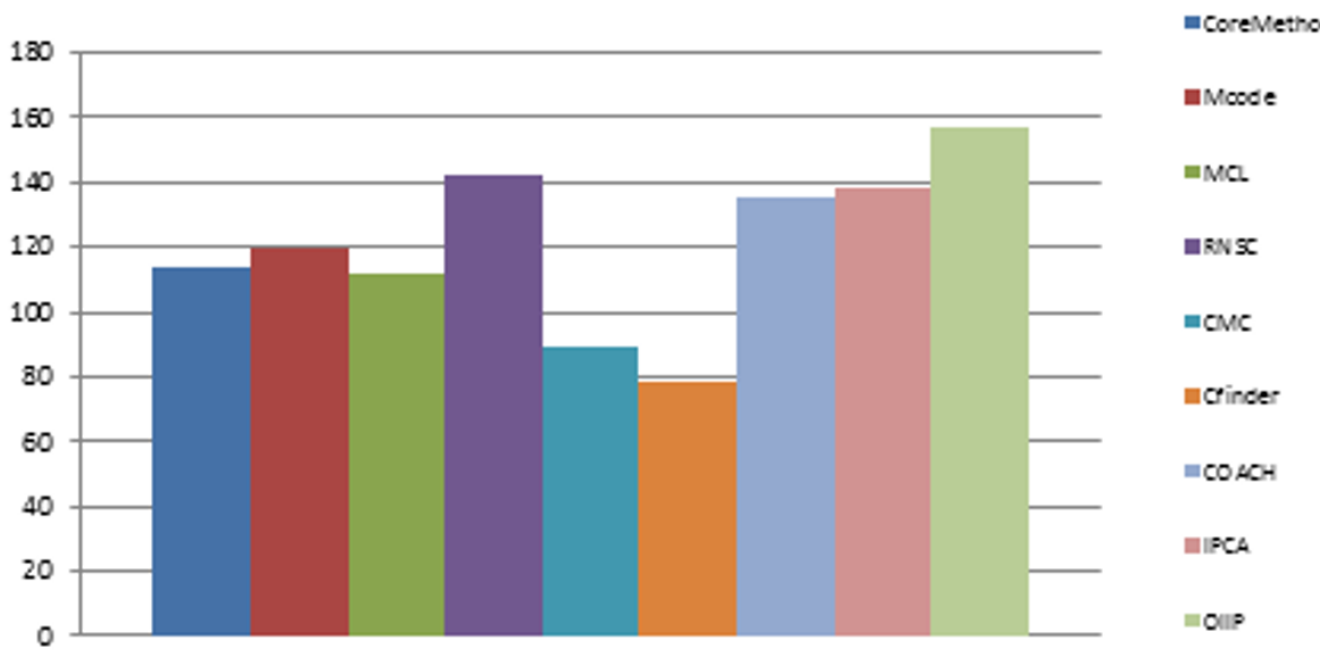

Figure 2 Comparison of the predicted clusters with the known complexes on Gavin PPI dataset. Figure 2 shows the numbers of known complexes (size>2) matched to the clusters generated by OIIP and by other eight previous known methods: CoreMethod[15], Mcode[9], MCL [13], RNSC[12], CMC[14], CFinder[11], COACH[10], IPCA[5].

network. When $\mathrm{T}_{\mathrm{in}}=0.5$, these Hub structures are decomposed into complexes that consist of only 2 proteins. Figure 1(b) shows that the size of the biggest cluster is decreasing as $T_{\text {in }}$ increases. With the increasing of $\mathrm{T}_{\mathrm{in}}$, the probability of neighbors added to the cluster is decreasing. Thus, the size of the predicted clusters is also decreasing.

As shown in Table 1, the Precision of the algorithm OIIP is about 0.6 when $T_{i n}=0.5$, which implies that the clusters generated by OIIP are reliable. The F-measure is about 0.45 when $\mathrm{T}_{\mathrm{in}}=0.4$, which represents overall performance of an algorithm. The Accuracy takes into account of both the sensitivity and PPV, and is determined by the larger one. In this experiment, the
Accuracy is mostly influenced by the PPV. The PPV of the clusters generated by OIIP increases with the increasing of $\mathrm{T}_{\mathrm{in}}$. Especially, an obvious increase appears when $T_{\text {in }} \geq 0.5$.

\section{Comparison of OIIP and other methods}

Since there have been protein complexes that were experimentally determined, a good protein complexes detecting algorithm should identify these known complexes as many as possible. Figure 2 shows the numbers of known complexes (size>2) matched to the clusters generated by OIIP and by other eight previous known methods: CoreMethod[37], Mcode[24], MCL[34], RNSC [33], CMC[36], CFinder[32], COACH[35], IPCA[14].

Table 2 Performance comparison of Identify protein complexes methods on Gavin dataset

\begin{tabular}{|c|c|c|c|c|c|c|c|}
\hline \multirow[t]{2}{*}{ Method } & \multirow[t]{2}{*}{ Precision } & \multirow[t]{2}{*}{ Recall } & \multirow[t]{2}{*}{ F-measure } & \multirow[t]{2}{*}{ Accuracy } & \multicolumn{3}{|c|}{ P-Value } \\
\hline & & & & & GO_Function & GO_Process & GO_Component \\
\hline CoreMethod & 0.526596 & 0.279412 & 0.365101 & 0.491702 & 0.579787 & 0.430851 & 0.478723 \\
\hline Mcode & 0.733333 & 0.294118 & 0.419847 & 0.469031 & 0.511111 & 0.392593 & 0.474074 \\
\hline $\mathrm{MCL}$ & 0.540373 & 0.27451 & 0.364071 & 0.509139 & 0.503106 & 0.391304 & 0.459627 \\
\hline RNSC & 0.400651 & 0.348039 & 0.372497 & 0.489759 & 0.332248 & 0.247557 & 0.309446 \\
\hline CMC & 0.608 & 0.218 & 0.3211 & 0.474 & 0.692 & 0.55 & 0.633 \\
\hline CFinder & 0.663 & 0.191 & 0.297 & 0.419 & 0.602 & 0.439 & 0.551 \\
\hline $\mathrm{COACH}$ & 0.524 & 0.331 & 0.406 & 0.49 & 0.656 & 0.525 & 0.61 \\
\hline IPCA & 0.526032 & 0.338235 & 0.411731 & 0.48789 & 0.719928 & 0.587074 & 0.666068 \\
\hline OIIP $\mathrm{T}=0.4$ & 0.559816 & 0.384804 & 0.456097 & 0.521424 & 0.697853 & 0.553681 & 0.636503 \\
\hline OIIP T=0.5 & 0.606195 & 0.335784 & 0.432177 & 0.490394 & 0.769912 & 0.639381 & 0.710177 \\
\hline
\end{tabular}




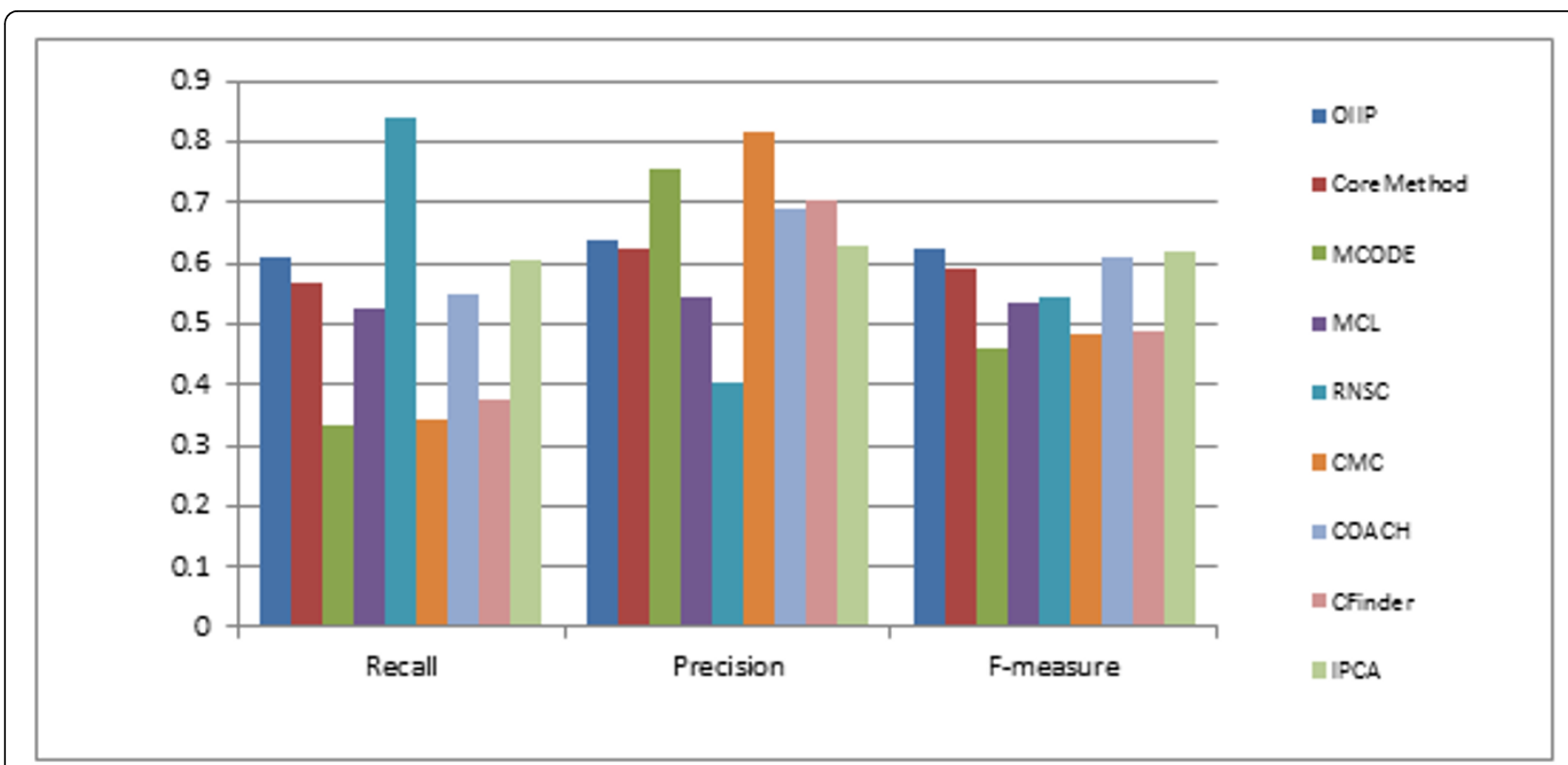

Figure 3 The Precision, Recall and F-value of results predicted from various methods on BioGrid PPI dataset. As show in Figure 3, RNSC has the highest recall and CMC has the highest precision, while our method OIIP also gets the highest F-measure on BioGrid PPI dataset.

Our method predicted more complexes than others on Gavin PPI dataset. As show in Table 2 OIIP also get the highest $\mathrm{F}$-measure $\left(\mathrm{T}_{\mathrm{in}}=04\right)$. Although Mcode has the highest precision, its recall is very low. The fact is that the predicted protein complexes are fewer than our method. Some predicted clusters which are not matched with criterion complexes are possible actual complexes which are undiscovered. So it is necessary to predict more clusters with high F-measure value.

And we count the number of clusters with p-value less than 0.01, a threshold which represents significant biological sense and compute the proportion of clusters

Table 3 Some predicted clusters which matched with benchmark complexes

\begin{tabular}{|c|c|c|c|c|c|c|}
\hline \multirow[t]{2}{*}{ ID } & \multirow[t]{2}{*}{ Predicted clusters } & \multirow{2}{*}{$\begin{array}{l}\text { Benchmark } \\
\text { complexes ID in } \\
\text { CYC2008 }\end{array}$} & \multirow[t]{2}{*}{ NA } & \multicolumn{3}{|c|}{ p-value } \\
\hline & & & & GO_Component & GO_Function & GO_Process \\
\hline 1 & YBR123C YOR110W YPL007C YDR362C YAL001C YGR047C & 402 & 1 & 0 & $3.16 \mathrm{E}-06$ & $2.59 \mathrm{E}-05$ \\
\hline 2 & YLR208W YGL092W YDL116W YJR042W YKL057C YGL100W & 181 & 1 & $1.10 \mathrm{E}-05$ & $2.50 \mathrm{E}-06$ & $3.81 \mathrm{E}-06$ \\
\hline 3 & $\begin{array}{c}\text { YLR166C YBR102C YPR055W YIL068C YER008C YDR166C YGL233W } \\
\text { YJL085W }\end{array}$ & 90 & 1 & $2.68 \mathrm{E}-06$ & $2.06 \mathrm{E}-04$ & $4.37 \mathrm{E}-03$ \\
\hline 4 & YBR234C YLR370C YJR065C YDL029W YIL062C YNR035C YKL013C & 12 & 1 & $1.19 \mathrm{E}-03$ & $4.95 E-06$ & $9.54 \mathrm{E}-07$ \\
\hline 5 & $\begin{array}{l}\text { YER157W YGR120C YPR105C YNL051W YML071C YGL223C } \\
\text { YNL041C YGL005C }\end{array}$ & 112 & 1 & $1.05 \mathrm{E}-05$ & $6.91 \mathrm{E}-02$ & $2.50 \mathrm{E}-06$ \\
\hline 6 & $\begin{array}{l}\text { YHR081W YHR069C YOL021C YGR095C YGR195W YDR280W } \\
\text { YGR158C YCR035C YDL111C YNL232W YOR001W YOL142W } \\
\text { YOR076C }\end{array}$ & 178 & 0.923077 & $3.00 \mathrm{E}-03$ & 0 & 0 \\
\hline 7 & $\begin{array}{c}\text { YGL048C YKL145W YHR027C YHL030W YLR421C YHR200W } \\
\text { YDR427W YDL147W YFR052W YDL097C YPR108W YIL075C } \\
\text { YFR004W }\end{array}$ & 2 & 0.911157 & $1.09 \mathrm{E}-05$ & $2.68 \mathrm{E}-06$ & 4.17E-06 \\
\hline 8 & $\begin{array}{l}\text { YPL210C YDL092W YML105C YPR088C YPL243W YDL051W } \\
\text { YKL122C }\end{array}$ & 248 & 0.857143 & $3.46 \mathrm{E}-06$ & 1.19E-07 & 1.19E-07 \\
\hline 9 & $\begin{array}{l}\text { YOR179C YDR195W YGR156W YER133W YAL043C YKL059C } \\
\text { YPR107C YLR115W YDR301W YNL317W YKR002W YKL018W } \\
\text { YLR277C }\end{array}$ & 341 & 0.816667 & $9.43 E-04$ & 0 & $2.32 \mathrm{E}-06$ \\
\hline 10 & $\begin{array}{l}\text { YMR223W YGL066W YBR081C YGR252W YDR448W YGL112C } \\
\text { YDR145W YMR236W YDR167W YBR198C YOL148C YLR055C } \\
\text { YPL254W YDR392W YCL010C YDR176W YHR099W YML007W }\end{array}$ & 227 & 0.802778 & 1.39E-04 & 0 & 0 \\
\hline
\end{tabular}


Table 4 Some predicted complexes which don't match with benchmark complexes

\begin{tabular}{|c|c|c|c|c|}
\hline \multirow[t]{2}{*}{ ID } & \multirow[t]{2}{*}{ Main proteins } & \multicolumn{2}{|r|}{ P-value } & \multirow[b]{2}{*}{ GO_Process } \\
\hline & & GO_Component & GO_Function & \\
\hline $\begin{array}{l}\text { Complex } \\
\text { a }\end{array}$ & $\begin{array}{l}\text { YOR201C YNL284C YCR046C YBL038W YDR237W YDR462W YDR296W YDR322W } \\
\text { YNL005C YMR024W }\end{array}$ & 9.89E-06 & $2.32 \mathrm{E}-06$ & $8.94 \mathrm{E}-07$ \\
\hline $\begin{array}{c}\text { Complex } \\
\text { b }\end{array}$ & YIL070C YNL137C YHL004W YPL013C YBR251W YDR041W YDR036C & 5.39E-05 & $8.58 \mathrm{E}-06$ & 4.95E-06 \\
\hline $\begin{array}{c}\text { Complex } \\
\mathrm{C}\end{array}$ & YNL112W YDR091C YMR309C YPR041W YBR079C YDR429C YOR096W YML063W & 0 & 2.03E-06 & $3.04 \mathrm{E}-04$ \\
\hline $\begin{array}{c}\text { Complex } \\
\mathrm{d}\end{array}$ & $\begin{array}{c}\text { YDR164C YOR204W YBL038W YML025C YGR220C YCR046C YNL005C YDR296W } \\
\text { YDR237W YNL284C YDR322W YDR462W YLR439W }\end{array}$ & $9.36 \mathrm{E}-06$ & $9.18 \mathrm{E}-06$ & $7.45 \mathrm{E}-06$ \\
\hline
\end{tabular}

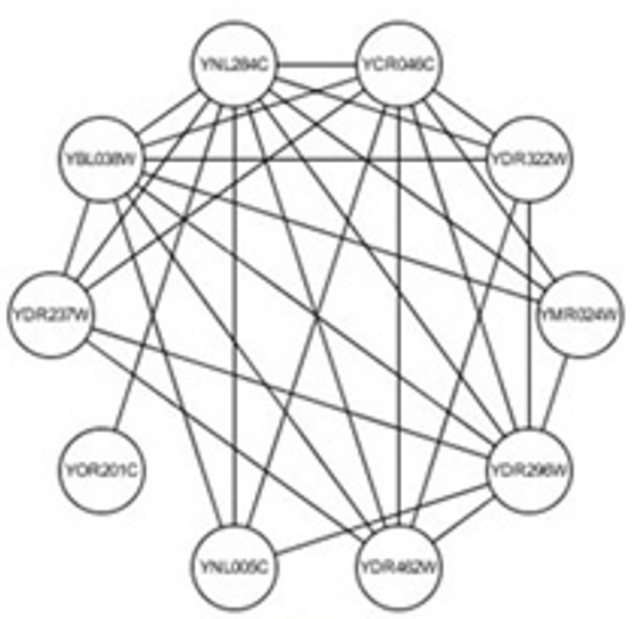

(a)

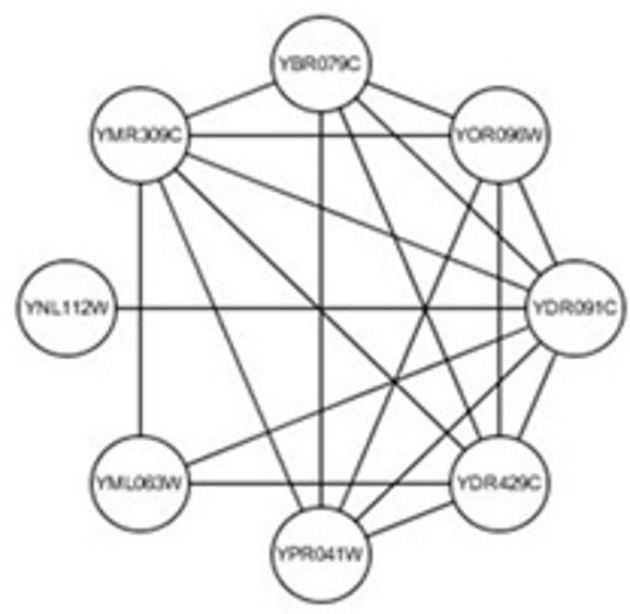

(c)

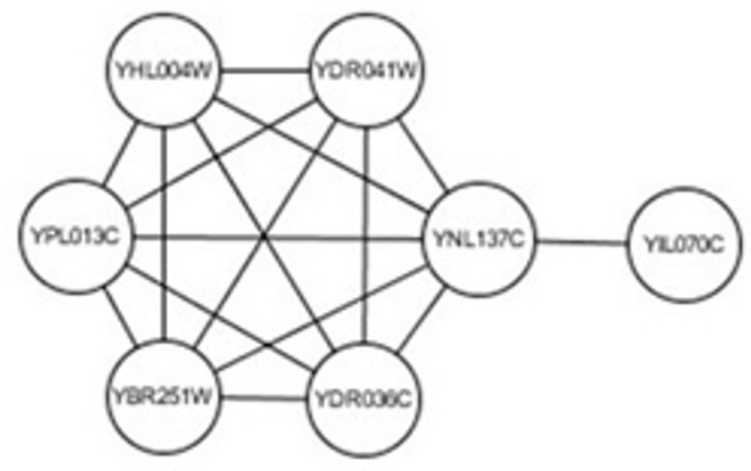

(b)

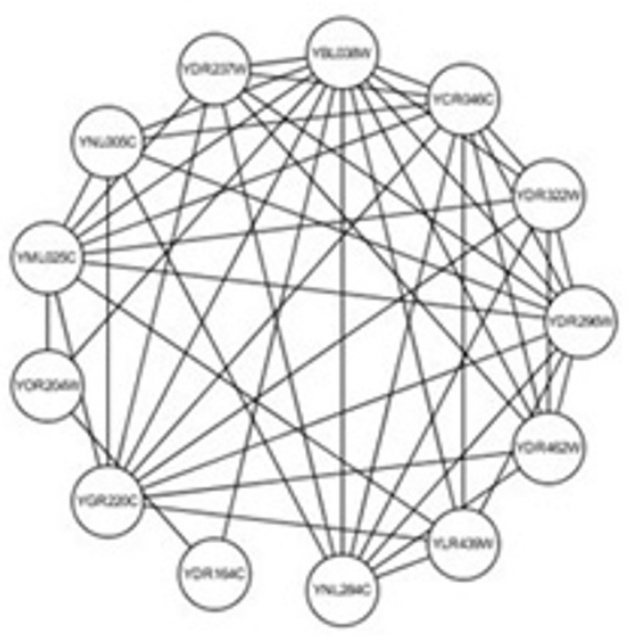

(d)

Figure 4 Some predicted complexes which don't match with benchmark complexes. Some of predicted clusters from our method are not matched with complex from criterion dataset. But we find that they have highly biologically significant and have high local density, so some of them may be real complexes which are still undiscovered. We also give some examples in Figure 4. Their p-value of biological annotation shows that some of them may be the candidate protein complexes. The results are useful for biologists to find the new protein complexes. 
which achieve low p-value. The proportion of clusters from various methods with low p-value is shown in Table 2. Table 2 also shows that the clusters predicted by our method have achieved highest biological significance than predicted clusters from others on all the three biological annotation datasets when $\mathrm{T}$ is set to 0.5 . Compare to the IPCA, we have better performance in all evaluation measurements. So the ontology interaction to the PPI network is valuable to predict protein complexes.

As show in Figure 3, RNSC has the highest recall and CMC has the highest precision, while our method OIIP also gets the highest F-measure on BioGrid PPI dataset.

\section{Examples of predicted complexes}

From the results of the experiment above we know that most of our predicted clusters have highly biological meanings. We give some examples of predicted clusters with detailed information which are matched with the benchmark complexes in Table 3 . We also list the best matched benchmark complexes, NA and the p-value of three protein annotation.

Some of predicted clusters from our method are not matched with complex from criterion dataset. But we find that they have highly biologically significant and have high local density, so some of them may be real complexes which are still undiscovered. We also give some examples in Table 4 and Figure 4 . Their p-value of biological annotation shows that some of them may be the candidate protein complexes. The results are useful for biologists to find the new protein complexes.

\section{Conclusions}

It is believed that identification of protein complexes is useful to explain certain biological progress and to predict functions of proteins. In this paper, we developed an algorithm OIIP to identify protein complexes based on the new large weighted protein interaction networks. Experimentally generated protein-protein interaction data includes an enormous amount of false positives. So we introduced a semantic similarity method to measure the reliability of interactions. For this measurement, we use the annotations in Gene Ontology (GO), which provides the comprehensive functional information. When we implemented the OIIP algorithm with weighted networks, the overall F-measure and accuracy of complexes is substantially improved. This result strongly appeals the necessity of integrating of functional information for the analysis of protein-protein interaction data.

The fact that biological properties are poor at the identification reveals that the higher-level structures (e. g., secondary and tertiary structure) of proteins cannot be accurately represented by the primary structure under the current coding techniques. The experimentally determined protein interaction network has not been used in the research, and a possible future research could combine the experimentally determined protein interactions with the GO estimated interactions to further improve the identification.

\section{Acknowledgements}

This work is supported by grant from the Natural Science Foundation of China (No. 60673039 and 61070098), the National High Tech Research and Development Plan of China (No. 2006AA01Z151), the Fundamental Research Funds for the Central Universities (No. DUT10JS09) and Liaoning Province Doctor Startup Fund (No. 20091015).

This article has been published as part of Proteome Science Volume 9 Supplement 1, 2011: Proceedings of the International Workshop on Computational Proteomics. The full contents of the supplement are available online at http://www.proteomesci.com/supplements/9/S1.

\section{Authors' contributions}

Please see sample text in BX identified all protein complexes and generated this manuscript; ZHY provided data information and edited this manuscript; HFL supervised all aspects of this manuscript. All authors read and approved the final manuscript.

\section{Competing interests}

The authors declare that they have no competing interests.

Published: 14 October 2011

\section{References}

1. Uetz P: A comprehensive analysis of protein-protein interactions in Saccharomyces cerevisiae. Nature 2000, 403(1):623-627.

2. Ito T: A comprehensive two-hybrid analysis to explore the yeast protein interactome. PNAS 2001, 98(8):4569-4574.

3. Gavin AC: Functional organization of the yeast proteome by systematic analysis of protein complexes. Nature 2002, 415(1):141-147.

4. Ho Y: Systematic identification of protein complexes in Saccharomyces cerevisiae by mass spectrometry. Nature 2002, 415(1):180-183.

5. von Mering C: Comparative assessment of large-scale data sets of protein-protein interactions. Nature 2002, 417(1):399-403.

6. Cho YR, Hwang W, Zhang A: Identification of overlapping functional modules in protein interaction networks: information flow-based approach. Proceedings of 6th IEEE International Conference on Data Mining Workshops 2006, 147-152.

7. Ideker T, Ozier O, Schwikowski B, Siegel AF: Discovering regulatory and signalling circuits in molecular interaction networks. Bioinformatics 2002, 18(1):S233-S240

8. Tornow S, Mewes HW: Functional modules by relating protein interaction networks and gene expression. Nucleic Acids Res 2003, 31(21):6283-6289.

9. The Gene Ontology Consortium: The Gene Ontology (GO) project in 2006. Nucleic Acids Research 2006, 34(1):322-326.

10. Hvidsten TR, Lagreid A, Komorowski J: Learning rule based models of biological process from gene expression time profiles using Gene Ontology. Bioinformatics 2003, 19(9):1116-1123.

11. Fang Z, Yang J, Li Y, Luo Q, Liu L: Knowledge guided analysis of microarray data. Journal of Biomedical Informatics 2006, 39(1):401-411.

12. Doherty JM, Carmichael LK, Mills JC: GOurmet: A tool for quantitative comparison and visualization of gene expression profiles based on gene ontology (GO) distributions. BMC Bioinformatics 2006, 7(151):332-365.

13. Barrat $A$, Barthelemy $M$, Pastor-Satorras $R$, Vespignani $A:$ The architecture of complex weighted networks. PNAS 2004, 101(11):3747-3752.

14. Li Min, Chen Jianer, Wang Jianxin: Modifying the DPClus algorithm for identifying protein complexes based on new topological structures. BMC Bioinformatics 2008, 9(398):345-352.

15. Shi C, Kaminskyj S, Caldwell S, Loewen MC: A role for a complex between activated $\mathrm{G}$ protein-coupled receptors in yeast cellular mating. Proc. Natl. Acad. Sci. U.S.A 2007, 104(13):5395-5400. 
16. Ortiz J, Stemmann O, Rank J: Lechner: A putative protein complex consisting of Ctf19, Mcm21, and Okp1 represents a missing link in the budding yeast kinetochore. Genes Dev 1999, 13(9):1140-1155.

17. Kraynack BA, Chan A, Rosenthal E, Essid M, Umansky B, Waters MG, Schmitt HD: Dsl1p, Tip20p, and the novel Dsl3(Sec39) protein are required for the stability of the Q/t-SNARE complex at the endoplasmic reticulum in yeast. Mol. Biol. Cell 2005, 16(9):3963-3977.

18. Chou KC, Cai YD: Predicting Protein-Protein Interactions from Sequences in a Hybridization Space. Proteome Res 2006, 5(1):316-322.

19. Ashburner M: Gene Ontology: tool for the unification of biology. Nat. Genet 2000, 25(1):25-29.

20. Chou JJ, Li H, Salvessen GS, Yuan J, Wagner G: Solution structure of BID, an intracellular amplifier of apoptotic signalling. Cell 1999, 96(1):615-624

21. Oxenoid $K$, Chou JJ: The structure of phospholamban pentamer reveals a channel-like architecture in membranes. Proc. Natl. Acad. Sci., U.S.A 2005, 102(1):10870-10875.

22. Resnik P: Using information content to evaluate semantic similarity in a taxonomy. The Proc. of 14th International Joint Conference on Artificial Intelligence 1995, 448-453.

23. Cho Y-R, Hwang W, Ranmanathan M, Zhang A: Semantic integration to identify overlapping functional modules in protein interaction networks. BMC Bioinformatics 2007, 265(8):147-160.

24. Bader G, Hogue C: An Automated Method for Finding Molecular Complexes in Large Protein Interaction Networks. BMC Bioinformatics 2003, 4(2):455-470

25. Chua HN, Ning K, Sung WK, Leong HW, Wong L: Using indirect proteinprotein interactions for protein complex prediction. CSB 2007, 2(1):97-109.

26. Geva G, Sharan R: Identification of Protein Complexes from Coimmunoprecipitation Data. Bioinformatics 2009, 74(3):1223-1146.

27. Wu M, Li XL, Kwoh CK, Ng SK: A Core-Attachment based Method to Detect Protein Complexes in PPI Networks. BMC Bioinformatics 2009 10(169):122-142.

28. Brohee S, van Helden J: Evaluation of clustering algorithms for proteinprotein interaction networks. BMC Bioinformatics 2006, 7(1):488-502.

29. Friedel CC, Krumsiek J, Zimmer R: Boostrapping the Interactome: Unsupervised Identification of Protein Complexes in Yeast. RECOMB 2008, 1(1):3-16.

30. Bu D, Zhao Y, Cai L, Xue H, Zhu X, Lu H, Zhang J, Sun S, Ling L, Zhang N, $\mathrm{Li} \mathrm{G}$, Chen R: Topological structure analysis of the protein-protein interaction network in budding yeast. Nucleic Acids Res 2003, 31(9):2443-2450

31. Gavin AC, Aloy P, Grandi P: Proteome survey reveals modularity of the yeast cell machinery. Nature 2006, 440(1):631-636.

32. Adamcsek B, Palla G, Farkas I, Derenyi I, Vicsek T: CFinder: locating cliques and overlapping modules in biological networks. Bioinformatics 2006, 22(8):1021-1043.

33. King A, Przulj N, Jurisica I: Protein Complex Prediction via Cost-based Clustering. Bioinformatics 2004, 20(1):3013-3020.

34. Pereira-Leal JB, Enright AJ, Ouzounis CA: Detection of functional modules from protein interaction networks. Protein Structure Function, and Bioinformatics 2004, 54(1):49-57.

35. Wu M, Li XL, Kwoh CK, Ng SK: A Core-Attachment based Method to Detect Protein Complexes in PPI Networks. BMC Bioinformatics 2009, 10(1):169-181.

36. Liu GM, Chua HN, Wong L: Complex discovery from weighted PPI networks. Bioinformatics 2009, 25(15):1891-1897.

37. Leung HC, Yiu SM, Xiang Q, Chin FY: Predicting Protein Complexes from PPI Data: A Core-Attachment Approach. Journal of Computational Biology 2009, 16(2):133-144.

38. Shuye Pu, Wong Jessica, Turner Brian: Up-to-data catalogues of yeast protein complexes. Nucleic Acids Research 2009, 37(1):825-831.

39. TG Consortium: The Gene Ontology Consortium: Creating the gene ontology resource: design and implementation. Genome Res 2001, 11(1):1425-1433.

40. Altaf-Ul-Amin M, Shinbo Y, Mihara K, Kurokawa K, Kanaya S: Development and implementation of an algorithm for detection of protein complexes in large interaction networks. BMC Bioinformatics 2006, 207(3):134-163.
doi:10.1186/1477-5956-9-S1-S7

Cite this article as: Xu et al:: Ontology integration to identify protein complex in protein interaction networks. Proteome Science 2011 9(Suppl 1):S7.

\section{Submit your next manuscript to BioMed Central and take full advantage of:}

- Convenient online submission

- Thorough peer review

- No space constraints or color figure charges

- Immediate publication on acceptance

- Inclusion in PubMed, CAS, Scopus and Google Scholar

- Research which is freely available for redistribution

Submit your manuscript at www.biomedcentral.com/submit
Biomed Central 